\title{
PSGL-1 Restricts HIV-1 Infectivity by Blocking Virus Particle Attachment to Target Cells ${ }^{\dagger}$
}

\author{
Yajing Fu ${ }^{1,2,3},{ }^{*}, \mathrm{Sijia} \mathrm{He}^{3}$, Abdul Waheed ${ }^{4}$, Deemah Dabbagh ${ }^{3}$, Zheng Zhou ${ }^{3}$, Benjamin Trinité ${ }^{5}$, Zhao \\ Wang ${ }^{6}$, Jieshi Yu ${ }^{6}$, Dan Wang ${ }^{6}$, Feng Li ${ }^{6}$, David N Levy ${ }^{5}$, Hong Shang ${ }^{1,2}$, Eric O Freed 4,* and Yuntao Wu ${ }^{3, *}$ \\ 1 Key Laboratory of AIDS Immunology of National Health Commission, Department of Laboratory \\ Medicine, The First Affiliated Hospital, China Medical University, Shenyang 110122, China; \\ hongshang100@hotmail.com \\ 2 Key Laboratory of AIDS Immunology, Chinese Academy of Medical Sciences, Shenyang 110001, China \\ 3 National Center for Biodefense and Infectious Diseases, School of Systems Biology, George Mason \\ University, Manassas, VA 20110, USA; she3@gmu.edu (S.H.); dabbagh.deemah@gmail.com (D.D.); \\ zzhou6@masonlive.gmu.edu (Z.Z.) \\ 4 Virus-Cell Interaction Section, HIV Dynamics and Replication Program, NCI-Frederick, Frederick, MD \\ 21702 USA; waheedab@nih.gov \\ 5 Department of Basic Science, New York University College of Dentistry, New York, NY 10010, USA; \\ bt32@nyu.edu (B.T.); dnlevy@nyu.edu (D.N.L.) \\ 6 Department of Biology and Microbiology, South Dakota State University, Brookings, SD 57007, USA; \\ zhaowang2007@outlook.com (Z.W.); jieshi.yu@sdstate.edu (J.Y.); dan.wang@sdstate.edu (D.W.); \\ feng.li@sdstate.edu (F.L.) \\ * Correspondence: fufu80s@sina.com (Y.F.), efreed@nih.gov (E.O.F.), ywu8@gmu.edu (Y.W.) \\ † Presented at Viruses 2020-Novel Concepts in Virology, Barcelona, Spain, 5-7 February 2020.
}

Published: 17 June 2020

\begin{abstract}
P-selectin glycoprotein ligand-1 (PSGL-1) is a dimeric, mucin-like, 120-kDa glycoprotein that binds to P-, E-, and L-selectins. PSGL-1 is primarily expressed on the surface of lymphoid and myeloid cells and is up-regulated during inflammation to mediate leukocyte tethering and rolling on the surface of endothelium for migration into inflamed tissues. Although it has been reported that PSGL-1 expression inhibits human immunodeficiency virus type 1 (HIV-1) replication, the mechanism of PSGL-1-mediated anti-HIV activity remains to be elucidated. Here, we report that PSGL-1 in virions blocks the infectivity of HIV-1 particles by preventing the binding of particles to target cells. This inhibitory activity is independent of the viral glycoprotein present on the virus particle; the binding of particles bearing the HIV-1 envelope glycoprotein, vesicular stomatitis virus $\mathrm{G}$ glycoprotein, or lacking a viral glycoprotein, is impaired by PSGL-1. Mapping studies show that the extracellular, N-terminal domain of PSGL-1 is necessary for its anti-HIV-1 activity, and the PSGL-1 cytoplasmic tail contributes to its inhibition. In addition, we demonstrate that the PSGL-1-related monomeric E-selectin-binding glycoprotein CD43 also effectively blocks HIV-1 infectivity. HIV-1 infection, or the expression of either Vpu or Nef, downregulates PSGL-1 from the cell surface; the expression of Vpu appears to be primarily responsible for enabling the virus to partially escape PSGL-1-mediated restriction. Finally, we show that PSGL-1 inhibits the infectivity of other viruses such as murine leukemia virus and influenza A virus. These findings demonstrate that PSGL-1 is a broad-spectrum antiviral host factor with a novel mechanism of action.
\end{abstract}

Keywords: virus release; innate immunity; antiviral factors

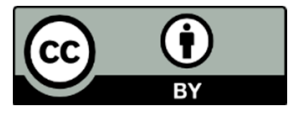

